Musées, Patrimoine et Culture scientifiques et techniques

$132 \mid 2010$

novembre - décembre 2010

\title{
La médiation écrite au musée : miroirs et jeux de miroirs
}

Written mediation in a museum context: mirrors and reflections

\section{Pascale Ancel}

\section{OpenEdition \\ Journals}

Édition électronique

URL : http://journals.openedition.org/ocim/378

DOI : 10.4000/ocim.378

ISSN : 2108-646X

Éditeur

OCIM

Édition imprimée

Date de publication : 1 novembre 2010

Pagination : $14-20$

ISSN : 0994-1908

Référence électronique

Pascale Ancel, « La médiation écrite au musée : miroirs et jeux de miroirs », La Lettre de l'OCIM [En

ligne], 132 | 2010, mis en ligne le 01 novembre 2012, consulté le 30 avril 2019. URL : http://

journals.openedition.org/ocim/378; DOI : 10.4000/ocim.378

Ce document a été généré automatiquement le 30 avril 2019.

Tous droits réservés 


\title{
La médiation écrite au musée : miroirs et jeux de miroirs
}

Written mediation in a museum context : mirrors and reflections

\author{
Pascale Ancel
}

1 En parcourant une exposition ce sont des objets, des œuvres d'art, des idées... et des textes que le visiteur découvre. Ces textes dits "informatifs" sont destinés à communiquer des connaissances sur ce qui est présenté. Ils ont pour support des étiquettes " classiques ", des fiches de salle, des panneaux, voire les murs. Ils peuvent être scientifiques, historiques, poétiques ou philosophiques. De nombreuses études s'intéressent aux usages que les visiteurs font de cette médiation écrite. Ces évaluations portent essentiellement sur la place qu'occupe cet écrit dans un dispositif d'accompagnement ou d'aide à la visite. Mais dans le cadre de ces évaluations on constate que le visiteur interrogé est amené également à exprimer indirectement ses attentes à l'égard des concepteurs d'exposition et à évoquer la façon dont il estime être considéré par les instances culturelles. En filigrane de ces écrits de médiation le visiteur-lecteur percevrait quelques unes des valeurs défendues par les institutions culturelles.

2 Trois recherches - consacrées chacune à l'évaluation d'expositions temporaires ou permanentes présentées à Grenoble et à Vizille - ont permis de mettre au jour certains aspects de l'usage des écrits médiatiques au moment des visites. La première étude était consacrée à l'exposition Le temps de la mélancolie, l'art italien et la Metafisica de 1912 à 1935, proposée par le musée de Grenoble en 2005. La deuxième étude effectuée au centre de culture scientifique, technique et industrielle La Casemate à Grenoble en 2007 concernait l'actualité scientifique et plus précisément la question des nanotechnologies. La troisième étude réalisée en 2008 au musée de la Révolution française de Vizille portait explicitement sur la médiation écrite de l'exposition permanente ${ }^{1}$.

3 Ces enquêtes ont été réalisées à partir d'entretiens semi-directifs. Ce type d'enquête qualitative permet d'accéder au sens que les personnes accordent à leurs pratiques, à leurs façons de voir et de vivre une situation précise afin d'en saisir toute la subjectivité. 
L'objectif de ces entretiens n'était pas de mesurer le niveau de connaissances des personnes interrogées dans les domaines artistique, scientifique ou historique mais de comprendre des expériences de visite en considérant la façon dont les visiteurs s'étaient approprié les textes, les images et les idées et la manière dont cela avait été mobilisé pendant et après la visite.

Pour traiter de la problématique de l'évaluation des textes à partir de ces études nous avons constitué un corpus de 107 entretiens. Ce sont les résultats transversaux de cette analyse thématique qui sont présentés ici. Pour chacune de ces études, le choix a été fait de constituer un échantillon diversifié, de nature contrastée, faisant varier au maximum les données « âge » et " profession » et veillant à une répartition équitable des femmes et des hommes. Tous les entretiens ont été réalisés auprès de visiteurs adultes, non captifs par opposition au public que les muséologues nomment les visiteurs "captifs", en particulier les scolaires - venus visiter l'exposition seuls ou en petits groupes (2 à 3 personnes) :

- l'étude réalisée au musée de Grenoble s'attachait à apprécier dans quelle mesure le caractère italianisant de cette exposition avait été un facteur déclenchant de visite. 14 personnes exprimant un lien avec l'Italie ont été interrogées. S'agissant d'une étude de réception de cette exposition sur la population d'origine italienne et sur les Italiens, la question de l'écrit et du texte était abordée parmi d'autres sujets ;

- Nanotechnologies : infiniment petit, maxi défis était la première exposition itinérante française d'information sur les nanotechnologies. Elle a été coproduite par le CCSTI La Casemate de Grenoble, la Cité des Sciences et de l'Industrie et Cap Sciences de Bordeaux. Le thème de l'exposition suscitant nombre d'interrogations, de réactions et parfois d'oppositions, l'étude de réception avait été menée dans l'objectif de saisir la place de ce type de manifestation dans la formation de l'opinion publique ${ }^{2}$. C'est dans ce cadre que des questions portant sur l'appréciation générale de la scénographie (comprenant des textes et de l'écrit) ont été posées. L'échantillon établi pour cette étude était composé de 43 personnes parmi lesquelles 24 ont déclaré une formation scientifique (au moins niveau bac). Il s'agissait d'un public que l'on pourrait qualifier d' «intéressé » - la démarche étant souvent volontaire, ils étaient venus spécialement pour cette exposition - mais non " spécialiste », seules quelques personnes se déclaraient réellement compétentes sur ce sujet scientifique ;

- l'étude menée au musée de la Révolution française de Vizille était précisément consacrée à l'appréciation des textes informatifs dans l'exposition permanente. 50 personnes ont été interrogées à la fin de leur visite. Il est important de préciser que le musée est hébergé dans un château classé monument historique, ancienne demeure des Ducs de Lesdiguières (XVIIe siècle) dans un parc d'une centaine d'hectares, lieu de détente privilégié des habitants de la région. La visite du château étant gratuite, elle s'inscrit parfois de façon improvisée dans le prolongement de la promenade.

Que pouvons-nous retenir de ces trois recherches ? Premièrement, les commentaires émis par les visiteurs révèlent que la plupart d'entre eux témoignent de compétences certaines en muséographie, révélant ainsi l'acquisition de ce que l'on peut appeler un « habitus muséographique ». Deuxièmement, cet habitus muséographique peut se comprendre au regard d'un horizon d'attentes, souvent lié au lieu d'exposition. Chaque établissement imprime un genre attendu, qu'il s'agisse d'une muséographie, d'un choix de points de vue ou de positions éthiques, qui sont connus et la plupart du temps appréciés des visiteurs. Cet ensemble d'éléments, de prises de positions et de manières de traiter un sujet 
participent d'une identité du lieu qui « conditionne » la visite. Troisièmement, dans ces écrits, les visiteurs trouvent certes des connaissances et des savoirs liés au thème de l'exposition, mais pas seulement. En effet, selon les personnes interrogées, la qualité et les contenus des écrits sont aussi de nature à rendre «lisibles » les intentions du musée à l'égard du visiteur. Ces écrits pourraient alors d'une certaine façon exprimer les valeurs culturelles au sens large portées par ces institutions.

\section{Un « habitus muséographique »}

6 Est-ce parce que les visiteurs sollicités dans ces études sont plutôt globalement diplômés que lorsqu'on les interroge sur les textes et sur leurs façons de les lire on découvre qu'ils ont beaucoup de choses à dire? De plus, lorsqu'ils font des suggestions c'est le plus souvent en comparaison avec des visites précédentes et/ou effectuées dans d'autres lieux. Nous pouvons considérer que ces visiteurs possèdent ce que nous avons appelé un "habitus ", c'est-à-dire un ensemble de dispositions durables et transposables. Ces savoirs incorporés sont souvent analysés par les visiteurs eux-mêmes au moment des entretiens.

7 Sans pouvoir ici entrer dans les détails, on remarque que les appréciations et les préconisations sur la médiation écrite concernent deux aspects. Dans un premier temps, les visiteurs s'attachent aux aspects formels et aux modalités de présentation des écrits, ils commentent les supports, s'interrogent sur des présentations originales, se félicitent de la quantité d'informations ou se déclarent fatigués par l'effort de lecture qui leur est demandé. Très souvent ce qui plait aux uns peut déplaire aux autres. Dans un second temps les commentaires concernent le niveau de lecture et les difficultés de la langue. Par exemple, les textes au contenu scientifique de l'exposition Nanotechnologies : infiniment petit maxi défis ont été remarqués, l'effort stylistique et la vulgarisation lexicale ont été soulignés et fortement appréciés par la majorité des visiteurs : «J'ai toujours un petit a priori par rapport à ce qui est scientifique par peur de ne pas comprendre, je ressors très satisfaite ", déclare cette responsable en transport maritime d'une quarantaine d'années. « Vu que je suis vraiment novice et que c'est assez abstrait pour moi tout ça, je trouve l'exposition assez claire ", confirme cette étudiante en droit.

8 Lors des entretiens, les commentaires font référence à ce qui a été vu et souvent apprécié ailleurs, confirmant que la familiarité avec les lieux de culture permet aux visiteurs de développer une véritable expertise d'usager : ils comparent les expositions entre elles, celles du même lieu qu'ils fréquentent régulièrement et celles des autres lieux qu'ils découvrent. C'est pourquoi ces visiteurs se sentent autorisés (même lorsqu'ils n'y sont pas invités par l'enquêteur) à donner leur avis sur l'exposition (livre d'or, échanges amicaux, blog...) et faire des rapprochements avec d'autres situations de visites, exercice auquel ils semblent se prêter avec un certain plaisir.

9 Parce qu'il développe une "culture " muséographique ce visiteur peut être qualifié d'expert. Mais veillons à ne pas réduire ce visiteur à son sens critique appliqué au seul domaine muséographique : ce « visiteur expert » n'est pas à strictement parler l'expert en art qui se rend au musée pour confirmer ses connaissances académiques, l'expert tel que nous l'entendons ici est ce visiteur capable d'apprendre à voir et à ressentir, par sa maitrise des codes de mise en exposition, ce qu'il ne connaissait pas avant d'avoir vécu au musée cette rencontre avec les œuvres et les discours sur les œuvres (Davallon, 
Gottesdiener, Poli, 2000). Autrement dit, le sens que le visiteur construit au cours et à l'issue de sa rencontre avec les œuvres est certes influencé par les énoncés dont il se saisit, mais il l'est tout autant par son approche personnelle de la visite. C'est ainsi par exemple que le cadrage historique et politique de l'exposition Le Temps de la mélancolie, l'art italien et la Metafisica de 1912 à 1935 a permis aux visiteurs d'origine italienne interrogés de replacer dans leur contexte les épisodes les plus intimes et les plus douloureux de leur histoire familiale, tel que l'exil par exemple.

Pour un grand nombre de personnes interrogées, la visite d'une exposition s'inscrit dans un ensemble plus vaste d'activités de connaissance ou de loisirs souvent liées à un contexte social ou professionnel. Ces pratiques que l'on peut qualifier de "culturelles " dans une large acception, contribuent à développer un type de compétences dans le domaine de la muséographie. Il n'est donc pas surprenant que les visiteurs, forts de ces expériences, se trouvent dans une disposition d'esprit particulière au moment de découvrir l'exposition.

\section{Un horizon d'attente}

11 La satisfaction des visiteurs est à mettre en regard avec l'idée d'horizon d'attente. Développée par Jauss dans le champ de la littérature, cette notion insiste sur le fait que lorsqu'une œuvre littéraire paraît, elle ne se présente jamais comme une nouveauté absolue : par tout un jeu d'annonces, de signaux - manifestes ou latents - de références implicites, de caractéristiques déjà familières, son public est prédisposé à un certain mode de réception.

Cette notion peut être rapportée aux cas des manifestations culturelles. Par exemple de nombreux visiteurs de l'exposition consacrée aux nanotechnologies déclaraient s'être rendus à « une proposition » du CCSTI, instance connue et fréquentée, dont ils déclarent apprécier la qualité du travail de vulgarisation scientifique et une certaine objectivité visà-vis des sujets traités. Cette proposition répondait à un certain nombre de normes attendues tant sur la forme que sur le fond. La reconnaissance de ces normes par les personnes interrogées a participé au sentiment de satisfaction éprouvé à l'issue de la visite : «je m'attendais à ça, à ce que ce soit bien détaillé pour que tout le monde puisse bien comprendre. Je m'attendais à des ateliers comme ça, à des explications, à des petits films pour qu'on en sache un peu plus. C'est très bien fait », confirme un logisticien d'affaires de 35 ans. Ce parti pris fort, indissociable du lieu d'exposition et de l'équipe de la Casemate, est souvent compris comme une signature et préviendrait d'une certaine façon contre le risque de déception.

13 Lors des entretiens réalisés à propos des textes expographiques on constate que c'est plus le lieu institutionnel qui bénéficie d'une forte reconnaissance que son responsable. Le conservateur ou le directeur de l'établissement concerné n'est jamais personnellement nommé, l'interlocuteur, celui qui s'engage et prend des risques (risque d'être contesté ou mal compris) c'est Le musée (de Grenoble), Le CCSTI ou Le château (de Vizille). Cependant l'horizon d'attente est largement satisfait dans la mesure où ce qui est proposé dans cet endroit devient pour le visiteur non pas un prêt à penser mais la possibilité de penser seul et à son rythme. La remarque de cette jeune étudiante en sociologie interrogée au CCSTI résume le sentiment général : «je prends ça comme quelque chose d'informatif, c'est-àdire que j'écoute et puis je me ferai une opinion après ». 
14 La plupart des visiteurs s'accordent sur le fait que les expositions présentées défendent un point de vue particulier qui participerait d'un "contrat de lecture», contrat qui s'établit entre le visiteur et le concepteur. Il ressort des analyses que plus le parti pris du conservateur est explicite et sans ambiguité, plus le visiteur se sent libre de ses lectures des textes. De façon paradoxale un cadre discursif, a priori contraignant n'enferme pas le visiteur dans une lecture imposée. Il permet au contraire à chacun de s'approprier l'exposition à des fins personnelles, lui offrant le choix des interprétations et des analyses du texte médiatique.

Dans le cas de La Metafisica, par exemple, la lecture historique proposée par le musée situait ce courant de la peinture dans le contexte social et politique de l'Italie du début du XXe siècle. Ainsi lorsqu'on demande aux visiteurs de rendre compte de leur visite en faisant référence aux œuvres exposées, on observe que ces interprétations font écho aux textes de l'exposition. Les références au fascisme et au contexte de l'époque étant largement explicitées dans le catalogue et sur les murs de l'exposition, il n'est pas surprenant que les visiteurs s'en soient inspirés pour construire leur relation aux tableaux. Mais ce cadre historique tel qu'il est écrit entre ensuite en résonance avec l'histoire personnelle : pour les personnes d'origine italienne les questions d'immigration sont toujours sensibles, la visite de l'exposition leur a permis de mettre au jour certains pans du passé familial en les replaçant dans un contexte politique et social, insistant sur la dimension historique de l'immigration. Grâce aux textes - pour la plupart présentés en thermo-collé sur les murs - les personnes s'étant de cette manière réapproprié quelques parcelles de leur histoire intime ont déclaré mieux comprendre le départ des parents : «mes parents sont arrivés en France dans les années 1920. C'est un peu cette époque là que les peintres décrivent. Je pense que la tristesse et l'austérité qui se dégagent de ces tableaux démontrent que ce n'était pas une période très simple en Italie. Donc c'est peut être un début d'explication des raisons pour lesquelles mes grands-parents ont quitté l'Italie », nous confie avec émotion cette éducatrice d'une cinquantaine d'années.

L'exposition consacrée aux nanotechnologies avait fait le choix d'inscrire un aspect scientifique et technique dans un questionnement de société abordant les aspects éthiques et philosophiques. Ce croisement des regards semble avoir séduit les visiteurs comme le résume cet étudiant: "ce qui m'a plu c'est d'avoir à la fois des avis de scientifiques et de philosophes et d'historiens des sciences sur ces questions ». Ce point de vue, qualifié d'objectif par le plus grand nombre et qui se prêtait à la confrontation de discours parfois contradictoires a été à la quasi-unanimité salué, certaines personnes bien que favorables à ces technologies, regrettant cependant que la parole des opposants les plus radicaux ne soit pas mieux diffusée comme l'exprime ce retraité : «j'ai besoin d'aller écouter les détracteurs aussi. Pour comparer, me faire ma propre idée ».

On peut retenir de ces études que la polyphonie énonciative - ce procédé d'écriture qui consiste à multiplier les points de vue et les perspectives - privilégiée dans ce type de médiation écrite a pour effet de faciliter l'appropriation personnelle des propos des concepteurs. Il semblerait que pour trouver sa place dans un dispositif narratif aux multiples facettes, le visiteur ait besoin d'identifier un parti pris fort comme point de départ de sa réflexion, soit pour s'y opposer, soit pour se laisser guider. 


\section{Le jeu de la réception} qualitatives éclairent les différentes façons dont la médiation écrite contribue à produire $\mathrm{du}$ sens pour le visiteur. Faire parler un visiteur sur la médiation écrite nous renseigne sur ses pratiques, mais permet également d'accéder d'une part aux représentations que celui-ci se fait du musée ou des autres lieux de culture et d'autre part à ses attentes à l'égard des institutions culturelles. Ainsi, les commentaires, remarques et critiques adressés aux textes concernent également leurs auteurs et l'institution culturelle à laquelle ils sont rattachés. Au-delà d'un contenu informatif, le lecteur semble lire dans ces écrits la nature des sentiments ou des valeurs (respect, indifférence...) que le conservateur, le commissaire ou le directeur porte sur son public.

Il ressort de l'analyse de ce corpus - recueilli dans trois établissements très différents mais à partir d'une même problématique - que, en tant que service public, le musée d'art ou tout autre lieu d'exposition est tenu de produire une variété d'écrits et de textes chargés de transmettre des connaissances et des savoirs experts. Les discours sur les politiques culturelles en France ont si bien fonctionné auprès des publics actifs que les lieux de culture sont perçus comme des dispositifs de démocratisation culturelle de haut niveau. Les visiteurs considèrent comme un droit légitime le fait qu'un lieu d'exposition propose à ses publics des écrits de médiation de qualité. Et parfois lorsqu'on les invite à proposer des améliorations certains, qui ont par ailleurs beaucoup de choses à dire, estiment que ce n'est pas à eux de les dire. Ainsi, lors d'un entretien au musée de la Révolution française, à la question : «Selon vous, quelles préconisations feriez vous pour améliorer les textes? » l'enquêteur s'est entendu répondre de façon cavalière : «Je ne sais pas moi. Y'a des gens qui ont bac + 5, c'est leur boulot !». Pour cette personne, employée de commerce de 47 ans, habitant la région et fréquentant le château et le parc depuis une vingtaine d'années, il est évident que la rédaction de ces documents relève de compétences particulières, reconnues, et ne peut donc pas être assurée par n'importe quel scripteur.

Pour les visiteurs, la qualité des énoncés révèle toute l'attention et la considération que le musée porte à son public. Il convient alors de trouver un juste équilibre entre un langage accessible à tous - qui ne provoque pas un sentiment d'indignité culturelle - et des informations trop vulgarisées - qui ne laissent pas le visiteur frustré - et donc d'offrir différents niveaux de lectures et de compréhension. Cet équilibre semble avoir été atteint lors de l'exposition La Metafisica où toutes les personnes d'origine italienne ont découvert dans les tableaux et dans les textes des résonances avec leur histoire personnelle. C'est aussi le cas de Nanotechnologies où les publics les plus différents (étudiants, scientifiques) ont estimé dans leur majorité que leur désir de découverte et de compréhension était assouvi. De même si pour quelques personnes interrogées l'identité du musée de Vizille reste indéfinie entre musée d'histoire, d'art ou de société, le fait de proposer plusieurs entrées de lecture n'est pas rédhibitoire. Chacun y trouve son compte selon ses préférences : histoire, art, artisanat... Sachant que tout ne sera pas lu, le visiteur se sent libre de ses choix.

21

Cette sensibilité ainsi exprimée rejoint l'idée que les concepteurs possèdent grâce aux mots un certain pouvoir qui serait celui de sélectionner, d'organiser les connaissances et donc d'orienter le regard. Si comme le déclare la plupart des personnes interrogées, ce 
point de vue est souvent attendu voire souhaité, ces dernières savent faire preuve de discernement au sujet des enjeux sociaux de transmission de la culture liés à ces textes encadrant les objets et les œuvres. Envisagé comme essentiel, le texte expographique n'est donc pas banalisé : si les habitués ont salué l'objectivité du CCSTI, ils ont estimé cependant que l'engagement des concepteurs n'était pas totalement neutre. Lors de ce type d'exposition scientifique portant sur des questions sensibles de société, il est admis que les concepteurs ne doivent craindre ni la pluralité des opinions ni la confrontation des points de vue les plus contradictoires.

\section{L'effet miroir}

S'il apparait évident que la lecture des écrits a pour objectif de trouver de l'information sur les sujets exposés, la nature et la qualité des textes pourront laisser envisager au visiteur les manières dont il se sent perçu par le concepteur d'exposition. Dans ce jeu de miroir, celui-ci cherche respect et considération, c'est donc l'ensemble des relations à la culture qui est en jeu dans ces textes et l'ensemble des intentions du musée qui est interprété. En filigrane le visiteur perçoit des valeurs revendiquées par les lieux culturels qu'il fréquente et qui s'expriment dans trois registres complémentaires :

- affirmer une volonté de démocratisation culturelle versus une politique élitiste : produire des textes accessibles, tout en ne sous-estimant pas les compétences du visiteur, présenter un ensemble de textes à différents niveaux de lecture ;

- accepter la fonction symbolique de l'art qui au travers des images et des œuvres exprime les grands thèmes du monde versus développer une fonction strictement pédagogique visant à ne transmettre que des contenus et des savoirs scientifiques. Une professeure de français d'une quarantaine d'années, dont les parents sont arrivés en France dans les années 1960 et à qui on demandait si sa présence dans l'exposition La Metafisica était liée à ses origines italiennes répond en ces termes: «Ces œuvres expriment l'angoisse de cette époque. C'est une angoisse liée à l'époque historique d'accord mais ce sont des sentiments assez universels et qui moi ne me renvoient pas du tout à mon italianité »;

- distribuer la parole et défendre des dispositifs muséographiques privilégiant des discours à plusieurs voix. Procédé remarqué au CCSTI mais également très apprécié au musée de Grenoble. Sur fond d'histoire, l'exposition faisait «dialoguer» les artistes avec les philosophes, De Chirico, de Filippo de Pisis, Schopenhauer, se cédant en quelque sorte mutuellement la parole. Ce photographe d'une cinquantaine d'années confirme le plaisir éprouvé à découvrir les propos des artistes : « Avoir la vision des artistes c'est toujours plus intéressant car les historiens d'art peuvent raconter beaucoup de choses mais quand on a la vision de l'artiste ça permet de se faire une idée plus précise de l'œuvre ».

L'évaluation des énoncés écrits participe à l'évaluation générale en termes de satisfaction d'une exposition. Les différentes modalités de réception des textes et de compréhension de l'ensemble du dispositif intéressent toujours le chercheur et les commanditaires des études. Mais les propos recueillis peuvent se révéler particulièrement riches lorsqu'il s'agit de découvrir les représentations que le visiteur se fait des institutions culturelles et de leurs intentions. En conclusion, dans ce jeu de miroirs, on notera tout autant la pertinence de bon nombre de remarques sur la complémentarité des différents supports langagiers $\mathrm{du}$ texte expographique dans les expositions (qu'elles soient artistique, historique ou scientifique), que la grande liberté avec laquelle les visiteurs s'approprient le registre textuel. 


\section{NOTES}

1. Des textes informatifs dans l'exposition permanente du musée comme discours institutionnel. Étude au musée de la Révolution Française de Vizille, Poli, M.-S. et Ancel, P., 2008.

Innovation et Opinion publique, Étude de réception de l'exposition scientifique Nanotechnologies : infiniment petit, maxi défis, centre de culture scientifique et technique, La Casemate, Grenoble, Poli, M.S. et Ancel, P., 2006.

Le temps de la mélancolie, l'art italien et la Metafisica de 1912 à 1935, Étude de fréquentation et de réception, musée de Grenoble, sous la direction scientifique de Marie-Sylvie Poli (Pascale Ancel, Yvonne Neyrat, Pierre Le Queau, Christian Surcouf), 2005.

2. Voir à ce sujet Ancel P. et Poli, M.-S. Opinion publique et nanotechnologies, la Lettre de l'OCIM, $\mathrm{n}$ • 118, juillet-août 2008, pp. 4-12.

\section{RÉSUMÉS}

À partir d'enquêtes réalisées dans trois musées de la région grenobloise, l'auteur analyse les différentes modalités de réception et de compréhension des textes par les visiteurs tout en constatant que cette évaluation va bien au-delà et permet d'abord de mesurer les attentes et les représentations des visiteurs et ensuite de s'interroger sur l'image que le musée souhaite donner à travers ces dispositifs textuels.

Stemming from surveys carried out in three museums in the area of Grenoble, the author analyses the different ways visitors receive and understand texts. She notes that this evaluation goes way beyond a simple analysis. Firstly, it allows the expectations and visitor representations to be measured, and secondly allows the image that the museum wishes to project through these textual means to be considered.

\section{INDEX}

Mots-clés : étude de publics, exposition, image, médiation, territoire, texte, visiteur Index géographique : France, Grenoble

\section{AUTEUR}

\section{PASCALE ANCEL}

Pascale Ancel est maître de conférences en Sociologie à l'université Pierre Mendès France de Grenoble. pascale.ancel@upmf-grenoble.fr 